\title{
Evaluación de la amniocentesis precoz en una institución brasileña de diagnóstico prenatal
}

\author{
Luz Mery Bernal Parra
}

1 Bióloga, Pontificia Universidad Católica Do Paraná. Magister en Biología Celular y Genética, Pontificia Universidad Javeriana. Doctorado en Biología Genética, Universidad De Sao Paulo. Directora de Genética y Biología Molecular Reproductiva, Universidad Nacional Abierta y a Distancia. Correspondencia: luz.bernal@unad.edu.co

\section{RESUMEN}

A pesar de que la amniocentesis tradicional (AT) es un procedimiento de comprobada seguridad y confianza, el diagnóstico citogenético resultante está disponible en un periodo tardío de la gestación. La amniocentesis realizada entre 12 y 14 semanas presenta algunas ventajas sobre la AT y otras relativas a la muestra de vellosidad corial (AVC), pero su seguridad requiere cuidadoso análisis. El objetivo de este estudio fue evaluar el riesgo de pérdida fetal y de anomalías congénitas en un grupo de 479 embarazadas (un único feto), sometidas a la amniocentesis precoz (12-14 6/7 semanas). Con procedimientos monitorizados con ultrasonografía se recolectó aproximadamente $1 \mathrm{ml}$ de líquido amniótico por semana de edad gestacional, la edad materna media fue de 35,7 \pm 4,58 años. La principal indicación para la realización del procedimiento fue edad materna de 35 años o más (un 66,1\%). Se realizó un seguimiento completo del 90,2\% (432/479) de las pacientes.

El líquido amniótico fue obtenido con éxito en la primera tentativa en 406 casos $(94,0 \%)$. La tasa de pérdidas fetales fue del $4,9 \%$, de ese porcentaje, un 2,9\% ocurrieron hasta la 28a semana. Las diferencias observadas para las tasas de pérdidas fetales en las diferentes edades maternas no fueron estadísticamente significativas, la distribución de pérdidas fetal por categoría de edad gestacional no presentó diferencias significativas. De las pacientes, un $5,1 \%$ y un $2,3 \%$ relataron pérdida de líquido amniótico y sangrado, respectivamente. Estos hallazgos estuvieron significativamente asociados a la pérdida fetal. Se observaron ocho casos $(2,0 \%)$ de problemas respiratorios y dos $(0,5 \%)$ de anomalías músculo-esqueléticas. Nuestros datos sugieren un aumento de complicaciones y pérdidas fetales en edades gestacionales más precoces, especialmente inferiores a 13 semanas. Con operadores expertos, cuidados técnicos y la minimización del volumen aspirado, la amniocentesis de 14 semanas presenta bajo riesgo de pérdida fetal o anomalías congénitas y puede ser ofrecida rutinariamente a las pacientes.

Palabras clave: amniocentesis precoz, pérdida fetal, anomalías congénitas.

\section{Amniocentesis early assessment in an institution of prenatal diagnosis in Brazil}

\section{ABSTRACT}

Although mid-trimester amniocentesis (MA) is a safety and reliability procedure, cytogenetic diagnosis report is only informed in an advanced period of pregnancy. First trimester amniocentesis 
(12-14 weeks) shows some advantages on MA and others of chorionic villus sampling (CVS), but its safety requires careful analysis. The aim of this study was to evaluate the fetal loss and congenital anomalies in a population of 479 pregnant women (single gestations) who underwent first trimester amniocentesis (12-14+6/7 weeks). Under ultrasound-guided procedures approximately $\mathrm{lml}$ of amniotic fluid per gestational week was collected. Mean maternal age was $35.7 \pm 1$ 4.58. The main indication for the test was maternal age 35 or greater (66.1\%). Follow up was successful in $90.2 \%(432 / 479)$ patients. Amniotic fluid was successfully aspirated in the first attempt in 406 cases (94\%). Fetal loss rate was $4.9 \%, 2.9 \%$ were observed before 28 weeks. Differences in fetal loss rates associated to different categories of maternal age were not statistically significant. The distribution of fetal losses by different categories of gestational age showed no significant differences. No association between fetal loss and collected volume of amniotic fluid was observed. $5.1 \%$ and $2.3 \%$ of patients reported amniotic fluid loss and bleeding, respectively. Such complications were significantly associated with fetal loss. Eight cases (2\%) of respiratory problems and two $(0.5 \%)$ of musculo-skeletal anomalies were observed. Our data suggest increased complications and fetal losses at earlier gestational ages, especially at less than 13 weeks. Amniocentesis at 14 weeks poses low risk of fetal loss or congenital anomalies and may be routinely offered to patients, provided that operators are experienced, technical measures taken, and collected volume of amniotic fluid is minimized.

Key words: early amniocentesis, fetal loss, congenital anomalies

Recibido: 01-06-2012 Aceptado: 09-05-2012

\section{INTRODUCCIÓN}

La amniocentesis tradicional (AT), realizada después de la semana quince de gestación, es la técnica más difundida para la obtención de muestras fetales para el estudio del cariotipo fetal. Este procedimiento presenta fácil realización y riesgo de pérdida gestacional alrededor del 1\% (1). Se cree que la extracción de 15 a $20 \mathrm{ml}$ de líquido amniótico no representa una reducción significativa del volumen total en esta fase de la gestación. Adicionalmente, la cantidad de células factibles recolectadas permite un buen crecimiento celular en cultivo. El principal problema de la AT es la edad gestacional en que los resultados son suministrados (17-18 semanas).

Cuando alguna aberración cromosómica es diagnosticada, la opción de la pareja en proseguir o no con el embarazo demanda rapidez; con el avance de la gestación, aumentan los riesgos quirúrgicos de una interrupción, así como los traumas psicológicos asociados (2).

El perfeccionamiento de la ultrasonografía obstétrica y de las técnicas de cultivo celular, así como la mayor experiencia de los especialistas en el diagnóstico prenatal viabilizó la amniocentesis antes de la semana quince de gestación (3). Esta modalidad ha alcanzado mayor relevancia, como se nota por el número creciente de trabajos publicados relatando las más diversas experiencias con las técnicas y las poblaciones estudiadas. Se considera que los resultados obtenidos en cuanto a calidad de las preparaciones citogenéticas en amniocentesis precoz (AP), realizada entre doce y catorce semanas gestacionales, son comparables a las de la AT (4).

Antes de doce semanas, las tasas de error en el cultivo y pérdida fetal asociados al procedimiento son más elevadas (5). En Brasil, la AP fue introducida en 1992 (6) 
y desde entonces ha sido rutinariamente ofrecida en el Instituto de Medicina Fetal y Genética Humana.

\section{MATERIALES Y MÉTODOS}

La población estudio consistió en embarazadas del Instituto de Medicina Fetal y Genética Humana de São Paulo. Antes del examen se verifica por ultrasonografía; la viabilidad fetal, edad gestacional, inserción de la placenta, el número de fetos y volumen de líquido amniótico. Se recolectó aproximadamente $1 \mathrm{ml}$ de líquido amniótico por semana gestacional, excepto en los primeros diecinueve procedimientos, realizados con aguja de veinte gauge, la punción fue realizada con aguja veintidós gauge bajo visualización ultrasonográfica directa. La punción con aguja veinte gauge fue abandonada por recomendación de Henry y Miller (7).

El procedimiento fué trasplacentário solamente cuando no había como puncionar otra región, no más de dos inserciones fueron realizadas el mismo día.Las intercurrencias relatadas fueron archivadas en la historia clínica. De este análisis, fueron excluidas las gestaciones múltiples. El líquido amniótico fue cultivado por la técnica de cultivo en frasco (8) en el laboratorio de Citogenética del Instituto.

Fueron analizadas 25 metafases por muestra: 10 con coloración Giemsa de rutina y 15 con bandas G. Técnicas adicionales de coloración (bandas C o NOR, por ejemplo) fueron realizadas cuando era necesario.

Después de la fecha probable de parto, fueron enviados cuestionarios a las pacientes, con la finalidad de tener información acerca de los datos obstétricos del parto y clínicos del recién nacido. El seguimiento de los casos fue realizado por teléfono (con la paciente o el obstetra) cuando esos datos no fueron remitidos por las pacientes.

La pérdida fetal fue definida como las pérdidas gestacionales espontáneas ocurridas después de la realización del examen. Estas fueron agrupadas en las siguientes categorías: 1. Hasta 30 días después del procedimiento; 2. Entre 31 días y 28 semanas gestacionales; 3. Por encima de 29 semanas gestacionales (inclusive natimortos y óbitos neonatales, hasta el trigésimo día de vida).

Las pruebas estadísticas consistieron fundamentalmente en el análisis de tablas de contingencia, generalmente reducidas las dimensiones 2x2, a las cuáles fue aplicada la prueba exacta de Fisher. En tablas 3x2 se empleó la prueba de qui-cuadrado, seguido por la prueba de Haberman (análisis de residuos ajustados) para localizar las clases responsables de la significancia, cuando esta tuvo lugar. En todas las pruebas se adoptó un nivel crítico de significancia estadística del $5 \%(\alpha=0,05)$.

El volumen medio de líquido amniótico obtenido a partir de gestaciones que resultaron en nativivos fue comparado con aquel obtenido en las gestaciones que resultaron en natimortos a través de la prueba $t$ de Student para el caso de varianzas de muestras heterogéneas.

Partos prematuros fueron aquellos ocurridos antes de las 37 semanas de gestación. Bajo peso al nacimiento se refirió a los recién nacidos con menos de 2.500g. El índice de Apgar $\left(1^{\circ}\right.$ y $5^{\circ}$ minuto de vida) fue el parámetro de evaluación de 
la vitalidad, fue analizado el hallazgo de anomalías congénitas.

\section{RESULTADOS}

Fueron incluidos en este estudio 479 procedimientos realizados en pacientes con gestaciones únicas del Instituto de Medicina Fetal y Genética Humana de São Paulo, entre junio de 1992 y agosto de 1998. En media la edad gestacional fue de 13,7 $\pm 0,69$ ( $m \pm 1$ d.p.) semanas y la edad materna de 35,7 + 4,58 años ( $m \pm 1$ d.p.). En el grupo por encima de 35 años, la edad media fue de 38,3 $\pm 2,41$ años. De las pacientes: $112(23,4 \%)$ eran primigestas; en 146 casos $(30,5 \%)$ había historia de aborto espontáneo; en $11(2,3 \%)$ de aborto por anomalías congénitas. Se observó placenta anterior, posterior, fúndica o lateral en $207(43,2 \%), 236(49,3 \%), 26(5,4 \%)$ y diez $(2,1 \%)$ pacientes, respectivamente. Las indicaciones para la realización del procedimiento están relacionadas en la tabla 1. La distribución de la edad gestacional en que los procedimientos fueron realizados en función de la edad materna se presentan en la tabla 2.

De las 479 gestaciones evaluadas se consiguió un seguimiento completo del 90,2\% (432/479). Los resultados fueron analizados con base en este total, tabla 2.

En 78 casos $(18,1 \%)$, el procedimiento fue transplacentário. En 406 casos (94,0\%), el líquido amniótico fue obtenido con éxito en la primera tentativa (una inserción) y en 26 casos (6,0\%), fueron necesarias dos tentativas. El volumen de líquido amniótico recolectado varió de 7 a $20 \mathrm{ml}(\mathrm{m} \pm 1 \mathrm{~d} . \mathrm{p} .=14,3 \pm 1,5 \mathrm{ml})$.

Tabla 1. Indicadores para la realización de la amniocentesis precoz

\begin{tabular}{|c|c|c|}
\hline INDICADORES & TOTAL & (\%) \\
\hline $\begin{array}{c}\text { Edad materna avanzada } \geq \mathbf{3 5} \text { años } \\
\text { • Sin otros indicadores }\end{array}$ & $\mathbf{3 1 7}$ & $\mathbf{6 6 . 1}$ \\
& $\mathbf{2 6 9}$ & $\mathbf{5 6 . 1}$ \\
\hline • Con otros indicadores & $\mathbf{4 8}$ & $\mathbf{1 0 . 0}$ \\
\hline Anomalía cromosómica previa & 6 & 1.3 \\
Hijo anterior con malformación congénita & 4 & 0.8 \\
Anomalía en ultrasonido & 5 & 1.0 \\
\hline Reordenamientos cromosómicos en & 2 & 0.4 \\
\hline los padres & 17 & 3.5 \\
\hline Anomalía cromosómica en VC en mosaico & 6 & 1.3 \\
\hline Anomalía cromosómica en VC sin mosaico & 8 & 1.7 \\
\hline Repetición de aborto & 104 & 21.7 \\
\hline Edad materna <35 años & 16 & 3.3 \\
\hline Ansiedad & 9 & 1.9 \\
Anomalía cromosómica previa & 8 & 1.7 \\
\hline Hijo anterior con malformación congénita & 8 & 1.7 \\
Anomalía en ultrasonido & 2 & 0.4 \\
\hline Anomalía cromosómica en VC en mosaico & 9 & 1.9 \\
Anomalía cromosómica en VC sin mosaico & 6 & 1.3 \\
\hline Anomalía cromosómica en VC en mosaico & $\mathbf{4 7 9}$ & $\mathbf{1 0 0}$ \\
\hline Anomalía cromosómica en VC sin mosaico & & \\
\hline TOTAL & & \\
\hline
\end{tabular}


La tasa de pérdidas fetales fue del 4,9\%. Nueve $(2,1 \%)$ pérdidas ocurrieron dentro de los 30 primeros días después de la realización del procedimiento y tres $(0,7 \%)$ hasta la semana 28 de gestación. La frecuencia de pérdidas gestacionales tardías (natimortos y óbitos neonatales) fue del 2,1\%.

En 17 casos (3,9\%) la interrupción fue voluntaria: una anormalidad detectada por ultrasonografía (anencefalia), un aborto social y quince aberraciones cromosómicas.

De estas últimas, se diagnosticaron: trisomía del cromosoma $21(\mathrm{n}=5)$, trisomía del cromosoma $18 \quad(n=2), \quad 47 . X X Y$ $(n=3), 47, X X X, 9 q h+(n=1), 46 . X Y / 47$. $\mathrm{XY}+18(\mathrm{n}=1), 46, \mathrm{XY} / 47, \mathrm{XY},+\operatorname{mar}(\mathrm{n}=1)$, $47, X X,+\operatorname{der}(22) t(17 ; 22)(p 13 ; q 11.2) m a t$ $(n=1)$ y $46, X X / 47, X X,+\operatorname{mar}(n=1)$.

Las diferencias observadas para la tasa de pérdida fetal, tabla 3 en diferentes edades maternas no son estadísticamente significativas (x2=0,376; 2 g,l.; $\mathrm{P}=0,829$ ), aún si se considera únicamente las pérdidas ocurridas en los primeros 30 días después del procedimiento (prueba exacta de Fisher $\mathrm{P}=0,158$ ).

Tabla 2. Número de procedimientos distribuidos por edad materna, edad gestacional y algunas informaciones clínicas sobre los procedimientos.

\begin{tabular}{|c|c|c|c|c|}
\hline \multicolumn{5}{|c|}{ Semanas de Gestación } \\
\hline $\begin{array}{l}\text { Edad materna } \\
\text { (años) }\end{array}$ & $\begin{array}{c}12-126 / 7 \\
n\end{array}$ & $\begin{array}{c}13-136 / 7 \\
n\end{array}$ & $\begin{array}{c}14-146 / 7 \\
n\end{array}$ & $\begin{array}{c}\text { TOTAL } \\
\mathbf{n}\end{array}$ \\
\hline$\leq 34$ & 18 & 82 & 58 & $158(33.0 \%)$ \\
\hline $\begin{array}{c}35-39 \\
\geq 40 \\
\text { TOTAL }\end{array}$ & $\begin{array}{c}18 \\
7 \\
\mathbf{4 3}(9.0 \%)\end{array}$ & $\begin{array}{c}113 \\
57 \\
\mathbf{2 5 2}(\mathbf{5 2 . 6 \% )}\end{array}$ & $\begin{array}{c}89 \\
37 \\
184(38.4 \%)\end{array}$ & $\begin{array}{l}220(46.0 \%) \\
101(21.0 \%)\end{array}$ \\
\hline Seguimiento & 36 & 231 & & 479 (100\%) \\
\hline $\begin{array}{l}\text { Medida vol. LA ( } m \pm 1 \text { d.p) } \\
\text { Insertos } 1\end{array}$ & $\begin{array}{c}13.5( \pm 1.8) \\
33\end{array}$ & $\begin{array}{c}14.0( \pm 1.3) \\
220\end{array}$ & $\begin{array}{c}165 \\
14.9( \pm 1.3) \\
153\end{array}$ & $\begin{array}{l}14.3( \pm 1.5) \\
406(94.0 \%)\end{array}$ \\
\hline $\begin{array}{c}\text { (n) } 2 \\
\text { Procedimiento } \\
\text { Transplacentario }\end{array}$ & $\begin{array}{l}3 \\
7\end{array}$ & $\begin{array}{l}11 \\
34\end{array}$ & $\begin{array}{l}12 \\
37\end{array}$ & $\begin{array}{l}26(6.0 \%) \\
78(18.1 \%)\end{array}$ \\
\hline No transplacentario & 29 & 197 & 128 & 354 (81.9\%) \\
\hline \multicolumn{5}{|c|}{ No transplacentario } \\
\hline \multicolumn{5}{|c|}{ LA: líquido amniótico } \\
\hline
\end{tabular}


La distribución de las pérdidas fetales por las tres categorías de edad gestacional, tabla 4, no presentó diferencias significativas $(\% 2=5,578 ; 2$ g.l.; $\mathrm{P}=0,061)$. La prueba exacta de Fisher paralas categorías de edad gestacional: 12-12 6/7 y 13-14 6/7 no fue significativa $(\mathrm{P}=0,401)$.

De acuerdo con la prueba exacta de Fisher $(\mathrm{P}=0,824)$, la pérdida fetal no varió entre las dos categorías de volumen de líquido amniótico recolectado, tabla 5 . El volumen medio de líquido amniótico obtenido en casos de nacidos vivos o en casos de natimortos (respectivamente 14,337 \pm 1,405 y $14,048 \pm 1,987$ ) reveló un resultado no significativo en la prueba $t$ de Student para varianzas heterogéneas $(\mathrm{t}=0,6578$; 21 g.L; P > 0,05).

Tabla 3. Aparición de pérdidas fetales de acuerdo con la edad materna

\begin{tabular}{|c|c|c|c|c|}
\hline & $\mathbf{n}$ & Nacidos vivos & Perdida fetal & Interrupción de gestación \\
\hline $\begin{array}{c}\text { Procedimientos con } \\
\text { seguimiento completo }\end{array}$ & 432 & $394(91,2 \%)$ & $21(4.9 \%)$ & $17(3.9 \%)$ \\
\hline $\begin{array}{c}\text { Edad materna } \\
\leq 34 \text { años }\end{array}$ & 143 & 136 & 7 & 0 \\
\hline $35-39$ años & & & & 7 \\
$\geq 40$ años & 201 & 185 & 9 & 10 \\
\hline \multicolumn{2}{|l}{} \\
& 88 & 73 & 5 & \\
\hline
\end{tabular}

Tabla 4. Frecuencia de pérdidas fetales en diferentes edades gestacionales

\begin{tabular}{|c|c|c|c|c|}
\hline & $\mathbf{n}$ & Nacidos vivos & Perdida fetal & $\begin{array}{c}\text { Interrupción de } \\
\text { gestación }\end{array}$ \\
\hline $\begin{array}{c}\text { Procedimientos con } \\
\text { seguimiento completo }\end{array}$ & 432 & $394(91,2 \%)$ & $21(4.9 \%)$ & $17(3.9 \%)$ \\
\hline $\begin{array}{c}\text { Edad materna } \\
12-126 / 7\end{array}$ & 36 & 31 & 3 & 2 \\
\hline $13-136 / 7$ & 231 & 208 & 15 & 8 \\
$14-146 / 7$ & 165 & 155 & 3 & 7 \\
\hline
\end{tabular}

Tabla 5. Frecuencia de pérdidas fetales por volumen de líquido amniótico aspirado

\begin{tabular}{|c|c|c|c|c|}
\hline & $\mathbf{n}$ & Nacidos vivos & Perdida fetal & $\begin{array}{c}\text { Interrupción de } \\
\text { gestación }\end{array}$ \\
\hline $\begin{array}{c}\text { Procedimientos con } \\
\text { seguimiento completo }\end{array}$ & 432 & $394(91,2 \%)$ & $21(4.9 \%)$ & $17(3.9 \%)$ \\
\hline
\end{tabular}




\begin{tabular}{|c|c|c|c|c|}
\hline & $\mathrm{n}$ & Nacidos vivos & Perdida fetal & $\begin{array}{c}\text { Interrupción de } \\
\text { gestación }\end{array}$ \\
\hline $\begin{array}{c}\text { Volumen de líquido amniótico } \\
<15 \mathrm{ml}\end{array}$ & 230 & 209 & 12 & 9 \\
$<15 \mathrm{ml}$ & 202 & 185 & 9 & 8 \\
\hline Texto exacto de Fisher $\mathrm{P}=0.824$ & & & & \\
\hline
\end{tabular}

De las pacientes, $22(5,1 \%)$ reportaron pérdida de líquido amniótico por vía vaginal después del procedimiento. En 18 $(81,8 \%)$ de esas pacientes, la intercurrencia se resolvió espontáneamente y el embarazo prosiguió hasta el término.

En las cuatro pacientes restantes $(18,2 \%)$, hubo aborto espontáneo, siendo dos de estos antes de 30 días después de la realización de la AP. Diez pacientes (2,3\%) presentaron sangrado. Cuatro de ellas abortaron espontáneamente, siendo dos dentro de los primeros 30 días después de la punción. En tres casos de esas pérdidas, además del sangrado, hubo pérdida de líquido amniótico.

La pérdida fetal fue significativa en gestaciones con pérdida de líquido amniótico (prueba exacta de Fisher, $\mathrm{P}=0,020$ ) $\mathrm{o}$ sangrado $(\mathrm{P}=0,001)$.
La pérdida de líquido amniótico (y2=1,094, 2 g.l., $\mathrm{P}=0,579)$ o sangrado $(\mathrm{x} 2=2,565$, 2 g.l., $\mathrm{P}=0,277)$ no varió significativamente en las diferentes edades gestacionales en que los procedimientos fueron realizados.

No fue significativa la diferencia observada entre la pérdida fetal en procedimientos transplacentários o no (Prueba exacta de Fisher: $\mathrm{P}=1,0$ ). Por otro lado, la realización de dos inserciones, tabla 6 , se asoció significativamente a la pérdida fetal (prueba exacta de Fisher: $\mathrm{P}=0,001$ ).

Nueve de las 432 gestaciones $(2,1 \%)$ fueron perdidas dentro de los 30 días subsecuentes a la realización del examen. Tres de esas nueve pacientes relataron complicaciones asociadas al procedimiento como pérdida de líquido amniótico, sangrado y cólico, las cuales no fueron observadas en las seis pacientes restantes.

Tabla 6. Frecuencia de pérdidas fetales en relación a las características del procedimiento

\begin{tabular}{|c|c|c|c|c|}
\hline & $\mathbf{n}$ & Nacidos vivos & Perdida fetal & $\begin{array}{c}\text { Interrupción de } \\
\text { gestación }\end{array}$ \\
\hline $\begin{array}{c}\text { Procedimientos con } \\
\text { seguimiento completo }\end{array}$ & 432 & $394(91,2 \%)$ & $\mathbf{2 1 ( 4 . 9 \% )}$ & $\mathbf{1 7 ( 3 . 9 \% )}$ \\
\hline $\begin{array}{c}\text { Procedimientos no } \\
\text { transpalcentarios }\end{array}$ & $354(81.9 \%)$ & 322 & $\mathbf{1 7}$ & $\mathbf{1 5}$ \\
\hline $\begin{array}{c}\text { Procedimientos } \\
\text { transplacentarios }\end{array}$ & $78(18.1 \%)$ & 72 & $\mathbf{4}$ & $\mathbf{2}$ \\
\hline Una inserción & 405 & 374 & $\mathbf{1 5}$ & $\mathbf{1 6}$ \\
\hline Dos inserciones & $27 \quad 20$ & $\mathbf{6}$ \\
\hline \multicolumn{2}{r|}{ Texto exacto de Fisher : $\mathrm{P}=1, \mathrm{P}=0.001$} \\
\hline
\end{tabular}


En la tabla 7, se resume el resultado perinatal. Las tasas de prematuridad y de bajo peso al nacimiento fueron del $9,4 \%$ y $8,4 \%$, respectivamente. Un índice de Apgar entre cero y tres en el $1^{\circ}$ minuto fue observado en cuatro $(1,0 \%)$ recién nacidos; catorce $(3,6 \%)$ recién nacidos presentaron Apgar entre cuatro y seis en el $1^{\circ}$ minuto; en dos $(0,5 \%)$, el índice entre cuatro y seis fue observado en el $5^{\circ}$ minuto, un Apgar entre siete y diez en el $1^{\circ}$ minuto fue observado en $376(95,4 \%)$ neonatos y en 392 $(99,5 \%)$ en el $5^{\circ}$ minuto. La patología neonatal más frecuente correspondió a ocho casos de problemas respiratorios $(2,0 \%)$; seis $(1,5 \%)$ de los cuáles necesitaron atención en la unidad de tratamiento intensivo neonatal (UTIN), después la evolución fue normal; se registraron también dos casos de anomalías músculo-esqueléticas (un torticolis congénito y una luxación congénita de la cadera).

\section{DISCUSIÓN}

Cerca de diez años atrás, las ventajas de la AVC como una opción precoz de diagnóstico prenatal fueron cuestionadas y muchas atenciones se volvieron a AP, considerada una alternativa atractiva, en vista de la fácil realización y de la satisfactoria cantidad de material recolectado para análisis citogenético. Una década después de haber sido introducida, la AP es aún controversial en lo que se refiere a riesgos y morbididad fetal. Algunos estudios reportan mayor riesgo asociado al procedimiento (9-12) mientras otros destacan ser la AP segura y una alternativa a los otros métodos de colecta (13-15). En los trabajos publicados los resultados son influenciados por las características de la población y la metodología del procedimiento.

Tabla 6. Frecuencia de pérdidas fetales en relación a las características del procedimiento

\begin{tabular}{|c|c|c|}
\hline Neonatos & $\mathrm{n}$ & $(\%)$ \\
\hline Total de procedimientos & 479 & 100 \\
\hline Seguimiento completo & 432 & 90.2 \\
\hline Nacidos vivos & 394 & 91.2 \\
\hline Nacimientos en un rango ( $\geq 37$ semanas) & 357 & 90.6 \\
\hline Nacimientos en un pre termino ( $<37$ semanas) & 37 & 9.4 \\
\hline Peso al nacer $\geq 2.500 \mathrm{~g}$ & 361 & 91.6 \\
\hline Bajo peso al nacer $(<2.500 \mathrm{~g})$ & 33 & 8.4 \\
\hline \multicolumn{3}{|l|}{ Anomalías en neonatos } \\
\hline Problemas respiratorios & 8 & 2.0 \\
\hline Problemas musculo-esqueléticos & 2 & 0.5 \\
\hline Anomalías cardiacas & 3 & 0.8 \\
\hline Otras* & 8 & 2.0 \\
\hline TOTAL & 21 & $5 \cdot 3$ \\
\hline Admitido por UTIN & $6^{* *}$ & 1.5 \\
\hline \multicolumn{3}{|c|}{$\begin{array}{l}\text { *Otras : cataratas congénitas, unión labio-palatino, hernia inguinal, hernia umbilical, hemangioma superciliar, } \\
\qquad \begin{array}{l}\text { hipospadia e hidrocele ( } 2 \text { casos) } \\
* * \text { todos por problemas respiratorios }\end{array}\end{array}$} \\
\hline
\end{tabular}


En el presente análisis, se reportan doce pérdidas gestacionales $(2,8 \%)$ desde la realización del procedimiento hasta la semana veintiocho de gestación y nueve pérdidas tardías $(2,1 \%)$ que consistieron en natimortos y óbitos neonatales. En total, la frecuencia de pérdidas fue del $4,9 \%$. Otros estudios encontraron frecuencias más bajas, situadas en tormo del 4\% (16), un $3,0 \%(17-19)$ o un $2,5 \%(5,20)$. Sin embargo, Schulman (21) encontró una frecuencia semejante (un 4,7\%) y Nagel (11) reportó una frecuencia mayor $(6,2 \%)$.

Evaluar la asociación entre las pérdidas fetales y los procedimientos invasivos en el diagnóstico prenatal requiere un trabajo arduo y no siempre satisfactorio. La alternativa más adecuada para esa tarea es la randomización. Como nuestra institución trabaja en ámbito privado, las pacientes no son receptivas al ingreso en un estudio que no permita la elección. La no randomización de los resultados, así como la inclusión de embarazadas de alto riesgo de pérdida gestacional en el estudio, debe haber influido en los resultados obtenidos.

De las pacientes con pérdida fetal: dos presentaban historia de aborto habitual; cuatro de hipertensión arterial; dos habían sido sometidas a otro procedimiento invasivo anteriormente (AVC) y una presentaba infección aguda por Citomegalovírus. Cada paciente con aborto habitual sufrió tres abortos anteriores. Las hipertensas presentaron eclampsia $(n=1)$ y pre-eclampsia $(n=3)$ después de la semana treinta.

Pacientes con historia de aborto habitual presentan mayor riesgo de pérdida gestacional subsecuente. El riesgo de aborto espontáneo para una embarazada con tres abortos anteriores llega a un 50\% (22), aunque otros estudios sitúan este valor entre 25 y un $45 \%(23,24)$.

Enfermedades infecciosas interfieren en el pronóstico de la gestación, cualquier infección severa podrá causar un aborto. La infección aguda por toxoplasmosis, citomegalovírus y herpes simple está asociada con aborto espontáneo (25).

La hipertensión arterial también se relaciona al mayor riesgo de pérdida gestacional. En un estudio colaborativo internacional, el grupo de pacientes hipertensas presentó un $11,1 \%$ de pérdida fetal mientras esa frecuencia fue del $2,6 \%$ en no-hipertensas, tal diferencia fue significativa (19).

Someter un embarazo a dos procedimientos invasivos puede aumentar el riesgo de pérdida fetal. Además de eso, la presencia de células citogenéticamente anormales en los tejidos extra-embrionarios puede influenciar la función placentaria. Esos factores implican pérdida fetal (26).

Otra explicación para la tasa de pérdida fetal encontrada sería la edad gestacional precoz en que el procedimiento fue realizado. Nuestros datos sugieren un aumento (aunque estadísticamente no significativo $\mathrm{P}=0,401)$ en la frecuencia de pérdidas fetales en las gestaciones más precoces, tabla 6, especialmente antes de 13 semanas. La frecuencia de pérdida gestacional decrece de un $8,3 \%(3 / 36)$ en la semana doce para un $6,5 \%(15 / 231)$ en la semana trece y un 1,8\% (3/165) en la semana catorce. $\mathrm{Al}$ agrupar los resultados de varias series de la literatura, las pérdidas fetales espontáneas se situaron alrededor del 6,2\% en 81 casos realizados entre las semanas diez y once de gestación y cayeron para un $2,3 \%$ en 1.558 casos realizados entre las 
semanas doce y trece $(5,7,13,17,27,28)$. En un estudio randomizado, la frecuencia de pérdida fetal espontánea fue también significativamente mayor en edades gestacionales más precoces: un $5,4 \%$ entre la semana diez y la once y un 4,0\% entre la semana doce y trece (9).

Una de las dificultades de los estudios dedicados a evaluar el riesgo de los procedimientos de colecta de tejidos fetales es la estimativa de las pérdidas basales, es decir, las pérdidas que ocurrirían independientemente del procedimiento. Para tanto, se utilizan datos referentes a pacientes que no han sido sometidas a ningún examen invasivo. Según Hanson (18), la tasa de aborto espontáneo después de la observación de resultados normales en examen ultrasonográfico puede oscilar entre un 2,1\% y un 3,2\% (antes de la semana catorce gestacional). Por otro lado, algunos estudios reportan una frecuencia mayor. Frates (29) encontró una frecuencia de pérdida fetal del $17 \%$ entre las semanas seis y nueve de gestación en mujeres saludables y con fetos viables y que no fueron sometidas a procedimientos invasivos. Esta tasa disminuyó para un $4,3 \%$ entre las semanas doce y trece.

En conjunto, los estudios relacionados encontraron una frecuencia de pérdida fetal de aproximadamente un 3,0\% en mujeres que no se sometieron a exámenes invasivos $(18,29-30)$. Analizando estos resultados, se concluye que por lo menos algunas de las pérdidas gestacionales subsecuentes a AP no dependerían del procedimiento.

En diversos manuscritos europeos y americanos, la frecuencia de pérdida fetal espontánea en los primeros treinta días después de la realización de AP es generalmente atribuida al procedimiento y oscila entre un $0,4 \%$ y un $2,3 \%(3,17,27,31)$. La frecuencia del $2,1 \%$ encontrada en nuestro estudio se encuentra en ese intervalo.

Es común observar en los trabajos publicados sobre AP mayor frecuencia de sangrado y pérdida de líquido amniótico que la observada en otros procedimientos. La incidencia de esas intercurrencias varía del $0,4 \%$ a un $5,4 \%$ y de el $1 \%$ a un $7,5 \%$, respectivamente $(7,10,11,12,18,20,31,32)$.

La ocurrencia de tales complicaciones es significante desde que una alta proporción de casos termine en pérdida fetal. En el presente estudio, se observó una frecuencia del 5,1\% de pérdida de líquido amniótico y del 2,3\% de sangrado. Ambas intercurrencias estuvieron significativamente asociadas a la pérdida fetal $(\mathrm{P}=0,020$ para la pérdida de líquido amniótico y $\mathrm{P}=0,001$ para sangrado). Así, las complicaciones asociadas a la técnica pueden ser una causa importante de la pérdida fetal.

El hecho de que las pérdidas fetales sean significativamente mayores en gestaciones con mayor cantidad de líquido amniótico extraído es un tema ampliamente discutido. En esta serie, el grupo con mayor tasa de pérdida fetal (un 4,6\%) se constituyó por casos en que se extrajo más de trece mililitros. Aunque el volumen de líquido amniótico recolectado no se haya relacionado estadísticamente con la frecuencia de pérdida fetal, debe existir una correlación entre el volumen recolectado y el pronóstico de la gestación. Mientras más precoz la gestación, más importante es la disminución del volumen total después de la retirada de una fracción de líquido amniótico para el estudio citogenético. El volumen de líquido amniótico habitualmente extraído en la semana dieciséis de 
gestación equivale aproximadamente a un $10 \%$ del total. Sin embargo, en las amniocentesis más precoces (aproximadamente doce mililitros entre la semana once y doce) ese volumen representa entre $20 \mathrm{y}$ un $25 \%$ del total de líquido amniótico. Se estima que la recuperación de la pérdida de líquido amniótico lleve de siete a diez días. La disminución brusca en el volumen de líquido amniótico y la descompresión temporal de la cavidad amniótica en la amniocentesis realizada antes de la semana trece de gestación pueden incrementar el riesgo de problemas respiratorios neonatales, malformaciones y aborto espontáneo $(33,34)$.

El aumento de la incidencia de problemas respiratorios en los neonatos cuyas madres se sometieron a AP es una preocupación constante. En nuestra serie fueron reportados ocho casos $(2,0 \%)$ de problemas respiratorios. Seis de estos necesitaron de atención en la unidad de tratamiento intensivo, con posterior evolución normal, y en ellos el volumen de líquido amniótico aspirado superó $13 \mathrm{ml}(\mathrm{m} \pm 1$ d.p. $=14,5 \pm$ 2,93). Conforme lo mencionado, la disminución brusca del volumen de líquido amniótico y la descompresión temporal de la cavidad amniótica en la edad gestacional de realización de AP, puede ser crítica para el desarrollo pulmonar. Una forma de minimizar el riesgo de problemas respiratorios sería la colecta de volúmenes próximos a $7 \mathrm{ml}$ (35).

La asociación entre AP y anomalías músculo-esqueléticas es otra preocupación. Dos casos $(0,5 \%)$ fueron observados: una luxación congénita de la cadera y un tortícolis congénito. A título de comparación, en una serie de 349 AP se relató $2,4 \%$ de luxación congénita de la cadera (19). Es interesante notar que, al contrario de otros trabajos sobre el mismo tema, no fueron observados casos de pie torcido congénito $(9,12,32)$. Esa anomalía es muchas veces atribuida a la descompresión de la cavidad amniótica subsecuente a la colecta y, a veces, a la pérdida de líquido amniótico (12).

En la población analizada en este estudio, las anomalías músculo-esqueléticas reportadas no estuvieron asociadas la pérdida de líquido amniótico. La remoción de menor cantidad de líquido amniótico en AP (de 3 la $6 \mathrm{ml}$ dependiendo de la semana gestacional) podría significar una disminución en la frecuencia de pérdida fetal y problemas ortopédicos y respiratorios en recién-nacidos (33).

Para algunos autores, la punción de la cavidad amniótica en gestaciones precoces es un procedimiento difícil, pues la pared uterina y las membranas ofrecen mayor resistencia. En algunos casos, más de una inserción es necesaria para poder obtener satisfactoriamente la muestra. Diaz (36), reporta una frecuencia relativamente alta de múltiples inserciones en los procedimientos precoces (7,7\%). Daniel (20), también presenta una frecuencia alta de múltiples inserciones $(12,9 \%)$ que fue asociada con una alta tasa de sangrado. Crandall (13) reporta una tasa mayor de múltiples inserciones en los procedimientos precoces $(4,0 \%)$ cuando comparados a los tradicionales (3,0\%). Diversos estudios demostraron que la frecuencia de pérdidas fetales aumentan en relación al número de inserciones en los procedimientos invasivos (37-39). En este trabajo, dos inserciones fueron necesarias en un 6,0\% de los casos, donde se observó una mayor frecuencia de pérdida fetal $(\mathrm{P}=0,001)$.

Cuando la placenta presenta inserción anterior, la punción puede ser transplacentária. 
Algunos autores sugieren una tasa mayor de complicaciones asociadas a ese tipo de procedimiento (40). Sin embargo, para otros autores la inserción anterior de la placenta y, consecuentemente, la punción transplacentária no aumenta la tasa de complicaciones cuando profesionales expertos realizan el procedimiento (41).

Las complicaciones asociadas a procedimientos transplacentários fueron contempladas en un estudio con 401 AP realizadas entre las semanas diez y la catorce de gestación. En 148 procedimientos transplacentários, hubo significativamente más casos de sangrado, aunque no se haya detectado diferencia en la tasa de pérdida fetal, pérdida de líquido amniótico y cólicos entre los grupos (34). En el presente estudio, no hubo correlación entre el procedimiento transplacentario y la ocurrencia de pérdidas fetales $(\mathrm{P}=1)$, sangrado $(\mathrm{P}=0,087)$ y pérdida de líquido amniótico $(\mathrm{P}=0,779)$.

Por lo tanto, AP transplacentária no parece aumentar los riesgos de pérdida fetal y complicaciones asociadas al procedimiento, conforme citado por Tharmaratnam (34).

La incidencia de prematuridad $(9,4 \%)$ y neonatos con bajo peso al nacimiento $(8,4 \%)$ en nuestra muestra es similar en relación la una población brasileña no sometida a exámenes invasivos en que la incidencia de ambas complicaciones se sitúa en tomo del $8,1 \%$ y un $9,5 \%$ respectivamente (42).

Después de la constatación de los hallazgos aquí relatados, en la institución donde fue realizado este análisis, la AP se tomó restricta a situaciones especiales. La técnica no se realizará en gestaciones con menos de trece semanas, pues además del riesgo asociado al procedimiento, hay un riesgo basal (ya aumentado en la población estudiada en función de la historia clínica).

Hay un esfuerzo en conseguir resultados a partir de cantidades cada vez más bajas de líquido amniótico. Hasta el momento, la mínima cantidad necesaria de líquido amniótico para la obtención de resultados citogenéticos fue fijada en aproximadamente diez mililitros en la semana trece.

Los centros que continúan ofreciendo AP en gestaciones más precoces, deben informar a las pacientes los riesgos de pérdida fetal y la incidencia de anomalías congénitas. Además de eso, cada institución debe contar con un registro de esas anomalías de modo a verificar una posible asociación con el procedimiento.

\section{REFERENCIAS}

1. Tabor A., Madsen M, Obel E.B., Philip J., Bang J., Noergard-Peterson B. (1986). Randomised controlled trial of genetic amniocentesis in 4606 low-risk women. Lancet i: 1287-1293.

2. Dallaire L., Lortie G., Des Rochers M., Clermont R., Vachon C. (1995). Parental reaction and adaptability to the prenatal diagnosis of fetal defect or genetic disease leading to pregnancy interruption. Prenat. Diagn., 15:249-259.

3. Hanson F.W., Zom E.M., Tennant F.R., Marionos S., Samuels S. (1987). Amniocentesis before 15 weeks gestation: Outcome, risks, and technical problems. Am. J. Obstet. GynecoI., 156:1524-1531.

4. Eiben B., Goebel R., Hansen S., Hammans W. (1994). Early amniocentesis. A cytogenetic evaluation of over 1500 cases. Prenat. Diagn., 14:497-501.

5. Elejalde B.R., de Elejalde M.M., Acuna J.A., Thelen D., Tiujillo C., Kamnann M. (1990). Prospective study of amniocentesis performed between weeks 9 and 16 of gestation: Its feasibility, risks, complications and use in early genetic prenatal diagnosis. Am. J. Med. Genet., 35:188-196. 
6. Gollop T.R., Naccache N.F., Pieri P.C. (1993). Amniocentese Precoce (12-15 semanas) com resultados rápidos: Uma realidade no Instituto de Medicina Fetal. Femina., 21:680-682.

7. Henry G.P., Miller W.A. (1992). Early Amniocentesis. J. Repmd. Med., 37(5):396-402.

8. Nadyr Fernandes Naccache, Instituto de Medicina Fetal y Genética Humana

9. Nicolaides K.H., Brizot M.L., Patel F., Snijders R.J. (1996). Comparison of Chorion Villus Sampling and Early Amniocentesis for karyotyping in 1492 singlenton pregnancies. Fetal. Diagn. Ther., 11:9-15.

10. Cederholm M., Axelsson O. (1997). A prospective comparative study on transabdominal chorionic villus sampling and amniocentesis performed at 10-13 weeks gestation. Prenat. Diagn., 17(4):311-317..

11. Nagel H.T.C., Vandenbussche F.P., Keirse M.J., Oepkes D., Oostewijk J.C., Beverstock G., Kanhai H.H. (1998). Amniocentesis before 14 completed weeks as an altemative to transabdominal chorionic villus sampling: A controlled trial with infant follow-up. Prenat. Diagn., 18:465-475.

12. The Canadian Early and Mid-Trimester Amniocentesis Trial (CEMAT) Group. (1998). Randomised trial to asses safety and fetal outcome of early and midtrimester amniocentesis. Lancet, 351:242-247.

13. Crandall B. F., Kulch P., Khalil T. (1994). Risk assessment of amniocentesis between 11 and 15 weeks: Comparison to later amniocentesis controls. Prenat. Diagn., 14:913-919.

14. Eiben B., Hammans W., Hansen S., Trawicki W., Osthelder B., Stelzer A., Jaspers KD., Goebel R. (1997). On the complication risk of Early Amniocentesis versus Standard Amniocentesis. Fetal. Diagn. Ther., 12:140-144.

15. Tavares P., Tavares A., Rendeiro P., Palmares C. (1998). Comparison between CVS and early amniocentesis. Prenat. Diagn., 18(1):87. Letters to editor.

16. Sato K., Izuta M., Kojima T., Miyakawa T., Yokoo I., Takahashi K, Tametika S., Okuno T., Saitou R., Toubai H. (1992). Early amniocentesis risk and laboratory evaluation. Acta.Obstet. Gynaecol. Jpn., 44:1285-1288.

17. Stripparo L., Buscaglia M., Longatti L., Ghisoni L., Dambrosio F., Guemeri S., Roselia
F., Lituania M., Cordones M., De Biasio P., Passamonti U., Gimeiii G., Cuoco C. (1990). Genetic amniocentesis; 505 cases performed before the sixteenth week of gestation. Prenat. Diagn., 10:359-364.

18. Hanson F.W., Tennant F.R, Hune S., Brookhyser K. (1992). Early amniocentesis: Outcome, risk, and technical problems at 12.8 weeks or less. Am. J. Obstet. GynecoI., 166:1707-1711.

19. Johnson J.M., Wilson R.D., Singer J., Winsor E., Harman C., Armson B.A., Benzie R., Dansereau J., Ho M.F., Mohide P., Natale R., Okun N. (1999). Technical factors in early amniocentesis predict adverse outcome. Results of the Canadiam Early (EA) versus Mid-trimester (MA) Amniocentesis Trial. Prenat. Diagn., 19:732-738.

20. Daniel A., NG A., Kuah K.B., Reiha S., Malafiej P. (1998). A study of early amniocentesis for prenatal cytogenetic diagnosis. Prenat. Diagn., 18(1):21-28.

21. Schulman L.P., Elias 8., Simpson J.L. (1991). Early amniocentesis: Complications in initial 150 cases compared to complications in initial 150 cases of transabdominal chorioric villus sampling. Am. J. Hum. Genet, 49:231.

22.Opitz, J.M. Tópicos recentes de genética clinica. (1984). Sociedade Brasileira de Genética Ribeirão Preto. São Paulo.

23. Boué J., Boué A., Lazar P. (1975). Retrospective and prospective epidemiological studies of 1500 karyotyped spontaneous human abortions. Teratology., 12:11-26.

24. Fitzsimmons J., Jackson D., Wapner R. Jackson L. (1983). Subsequent reproductive outcome in couples with repeated pregnancy loss. Am. J. Med. Genet., 16:583-587.

25. Stray-Pederson B., Stray-Pederson S. (1984). Etiologic factors and subsequent reproductive performance in 195 couples with a prior history of habitual abortion. Am. J. Obstet. Gynecol., 148:140-146.

26. Goldberg J.D., Porter A.E., Golbus M.S. (1990). Current assessment of fetal losses as a direct consequence of chorionic villus sampling. Am. J. Med. Genet, 35:174-177.

27. Nevin J., Nevin N.C., Doman J.C., Sim D., Armstrong M.J. (1990). Early amniocentesis: Experience of 222 consecutive patients, 19871988. Prenat. Diagn., 10:79-83. 
28. Schulman L.P., Elias 8., Phillips O.P., Grevengood C., Dungan J.S., Simpson J.L. (1994). Amniocentesis performed at 14 weeks gestation or earlier. Comparison with first-trimester transabdominal chorionic villus sampling. Obstet. Gynecol., 83:543-548.

29. Frates M.C., Benson C.B., Doubilet M. (1993). Pregnancy outcome after a first trimester sonogram demonstrating fetal cardiac activity. J. Ultrasound. Med., 12:383-386.

30. Wilson R.D., Kendrick V., Wittmann B.K., McGiIIivray B.C. (1986). Spontaneous abortion and pregnancy outcome after normal firsttrimester ultrasound examination. Am.J.Obstet. Gynecol., 67:352-355.

31. Brumfield C.G., Lin S., Conner W., Cosper P., Davis R.O., Owen J. (1996). Pregnancy outcome following genetic amniocentesis al $11-14$ versus 16-19 weeks gestation. Obstet. Gynecol., 88(1): 114-118.

32. Sundberg K., Bang J., Smidt-Jensen 8., Brocks V., Lundsteen C., Pamer J., Keiding N., Philip J. (1997). Randomised study of risk of fetal loss related to early amniocentesis versus chorionic villus sampling. Lancet., 350:697-703.

33. Eiben B., Hammans W., Trawicki W., Goebel R. (1998). Early Amniocentesis versus Chorionic Villus Sampling. Prenat. Diagn., 18:405-406, Letters to the editor.

34. Tharmaratnam S., Sadek S., Steele E.K., Harper M.A., Stewart F.J., Nevin J., Nevin N.C., Doman J.C. (1998). Early amniocentesis: Efect of removing a reduced volume of amniotic fluid on pregnancy outcome. Prenat. Diagn., 18:1773-778.

35. Tharmaratnam S., Sadek S., Steele E.K., Harper M.A., Nevin N.C., Doman JACI (1998). Transplacental early amniocentesis and pregnancy outcome. Br. J. Obstet. Gynaecol., 105(2):228-230.

36. Diaz M., De la Cueva P., Leal C_, Aisa F. (1996). Early Amniocentesis at $10-12$ weeks gestation. Prenat. Diagn., 16:307-312.

37. Aula P., Karjalinen 0., Terano N., Vaara L., Seppala M. (1979). Safety and accuracy of midtrimester amniocentesis for prenatal diagnosis of genetic disorders. Ann. Clin. Res., 11:156-159.

38. Simpson N., Dallaire L., Miller J., Siminovich L., Hammnerton J., Miller J., McKeen C. (1976). Prenatal diagnosis of genetic disease of Canada; report of a collaborative study. Can. Med. Ass. J., 115:739-745.

39. Reece E.A. (1997). Amniocentese genética precoce e nos trimestres intermediarios. Segurança e resultados. In: Clinicam Obstétricas e Ginecológicas da América do Norte. Diagnóstico e Tratamento Fetal. Reece E.A. Vol 1. Interlivros. Rio de Janeiro.

40. Mennuti M., Brummond W., Criombleholme W., Shwartz R. (1980). Fetal maternal bleeding associated with genetic amniocentesis. Obstet. GynecoI., 55:48.

41. Zolnierczyk P., Raczynski A., Lisawa J., Chazan B. (1997). Early amniocentesis transplacental needle passage safer than nontransplacental? Acta. Obstet. Gynecol. Scand., Supplement, 76(167):51.

42. DATASUS. (1997). Banco de dados do Sistema Único de Saúde. Disponível na Internet. http://www.datasus.saude.gov.br marco 2000. 